\title{
Survival study and clinicopathological evaluation of trichilemmal carcinoma
}

\author{
SHI-MIN ZHUANG ${ }^{1 *}$, GE-HUA ZHANG ${ }^{1 *}$, WEN-KUAN CHEN ${ }^{2}$, SHU-WEI CHEN ${ }^{2}$, \\ LI-PING WANG ${ }^{2}$, HUAN $\mathrm{LI}^{2}$ and MING SONG ${ }^{2}$
}

\author{
${ }^{1}$ Department of Otolaryngology-Head and Neck Surgery, The Third Affiliated Hospital Sun Yat-sen University, \\ Guangzhou, Guangdong 510630; ${ }^{2}$ Department of Head and Neck Surgery and State Key Laboratory of Oncology \\ in South China, Sun Yat-sen University Cancer Center, Guangzhou, Guangdong 510060, P.R. China
}

Received August 19, 2012; Accepted January 11, 2013

DOI: $10.3892 /$ mco.2013.74

\begin{abstract}
Trichilemmal carcinoma (TC) is a rare malignancy, commonly located on the scalp, forehead and neck, trunk or the upper extremities. The aim of this study was to investigate the clinicopathological characteristics and prognostic factors of TC, and to determine an optimal treatment strategy for these patients. Consecutive patients who were admitted to the Sun Yat-sen University Cancer Center between 1998 and 2012 were included in this retrospective study. The key prognostic factors affecting survival were lymph node metastasis and surgery margin. Multivariate analysis demonstrated that there was no risk factor for patient survival. Surgery margin and lymph node metastasis were the prognostic factors that influenced the treatment outcome. Simple excision with $1 \mathrm{~cm}$ margins is safe, cost-effective and effective for the treatment of TC. Additionally, postoperative follow-up of the patient in order to facilitate early diagnosis of recurrence and distant metastasis is necessary. Systemic chemotherapy should be considered in the case of patients with distant metastases.
\end{abstract}

\section{Introduction}

Trichilemmal carcinoma (TC) is a rare malignancy, commonly located on the scalp, forehead and neck, trunk or the upper extremities (1). It is known to develop from the external root sheath of the hair follicle, affecting predominantly the sun-exposed areas of the skin. (1). TC is also characterized by an indolent clinical course, and is usually considered to be curable

Correspondence to: Dr Ming Song, Department of Head and Neck Surgery and State Key Laboratory of Oncology in South China, Sun Yat-sen University Cancer Center, 651 Dongfeng Dong Road, Guangzhou, Guangdong 510060, P.R. China

E-mail: songming@sysucc.org.cn

\section{${ }^{*}$ Contributed equally}

Key words: trichilemmal carcinoma, locoregional recurrence, lymph nodes metastasis, treatment strategy, chemotherapy following surgical treatment in the form of wide surgical excision (2). TC clinical characteristics are usually not taken into consideration, while an onset of an abrupt growth phase leads to medical evaluation in the late stage of disease progression (3). A limited number of studies investigating TC are available, thus, the tumor behavior and prognosis are difficult to be estimated (4). Although TC has a low metastatic potential and does not usually recur, no long-term follow-up data have been collected on patients with this neoplasm, rendering deep invasion and local recurrence possible (5). In addition, no optimal treatment for TC with metastasis has been suggested and no established chemotherapy regimen for metastatic TC has been reported in the medical literature. Therefore, TC patients with metastasis have a poor prognosis (6). Although supported by limited data, the risk of recurrence and lymph node metastasis is potentially similar to that of squamous cell carcinoma (4).

However, since most of the previously published studies are case reports, only a limited number of studies have investigated the potential treatment strategies for this unique population (1,3-5). In the present study, to investigate this aspect, we performed a retrospective analysis of data obtained from the Sun Yat-sen University Cancer Center (Guangzhou, China). We evaluated the survival rate, clinical features and prognostic factors in 26 patients who developed TC. The aim of this study was to comprehensively identify the optimal therapeutic strategy for TC.

\section{Patients and methods}

Patient data. This retrospective study comprised consecutive patients admitted to the Sun Yat-sen University Cancer Center between February, 1998 and February, 2012, who were diagnosed with TC. Informed consent was obtained from the patients, while ethics approval was obtained from the Institutional Research Ethics Committee of the Sun Yat-sen University Cancer Center. In total, 26 TC patients were eligible to be included in this study (12 males and 14 females). The male/female ratio was 0.85 . The average age of patients with $\mathrm{TC}$ was 42.5 years.

The cumulative risk of the TC patients was estimated using the Kaplan-Meier method. Cox's proportional-hazard regression model was used to estimate the relative risk of survival 
following treatment. $\mathrm{P}<0.05$ was considered to indicate a statistically significant difference.

Clinical data. Most of the TC patients included in this study had a history of significant lifetime sun exposure. The pathological diagnosis of the patients was confirmed to be TC. Patients (12/26) were initially treated elsewhere and presented at the reported center at the time of the locoregional recurrence development. The most common tumor sites were areas of sun-exposed skin, including 14 cases located on the occipitalis, 6 on the scalp, 2 on the temporal region, 2 on the ear, 1 on the napex and 1 on the lower lip. Macroscopically, the neoplasms were described as exophytic, polypoid, ulcerated or nodular lesions, with or without malodor or bleeding. The size of the tumors ranged from 1 to $8 \mathrm{~cm}$ (mean size, $2.8 \mathrm{~cm}$ ). Twenty cases were characterized by non-invasive, and 6 cases by invasive boundary. In most of the TC patients, the lesion was present for a long time prior to diagnosis and a recent rapid growth phase occurred.

Follow-up data were collected from the outpatient service and complementary data were obtained by telephone inquiry and follow-up letters. The follow-up deadline was set for February 29, 2012. The median follow-up period was 63.8 months (range, 1-147 months).

Treatment. The patients were treated with surgery alone, and complete surgical excision with documented non-invasive intra- and postoperative margins were recommended for the patients. Eleven patients underwent direct suture after surgery, five reconstruction with rotation flap, and the remaining 10 patients underwent local rotation flap reconstruction and skin-grafting. Split skin was obtained from the abdomen (7 cases) and anterolateral (3 cases), meshed 1:1.5, and transplanted onto the dermal template. Postoperative course was uneventful for the patients. However, two patients who underwent expand resection and neck dissection developed lymph node metastasis and far recurrence after the initial surgery. Patients received chemotherapy treatment after surgery.

Statistical analysis. Univariate analysis was conducted using the Kaplan-Meier method and log-rank test. $\mathrm{P}<0.05$ was considered to indicate a statistically significant difference.

\section{Results}

Four of the 26 patients succumbed to the disease. The overall 5 -year survival rate of the patients was $89.2 \%$. Univariate analysis demonstrated that the key prognostic factors influencing the survival of these patients were lymph node metastasis and surgery margin (Figs. 1 and 2). There was no statistically significant correlation between TC and gender, tumor size, age and locoregional recurrence (Table I).

In the Cox proportional hazard regression model, there was no risk factor affecting the survival of TC patients.

\section{Discussion}

TC is a rare, cutaneous adnexal malignant tumor deriving from the outer root sheath of hair follicles usually affecting the sun-exposed areas of the skin in elderly people $(1,7)$. TC
Table I. Effect of clinicopathological characteristics on the survival of 26 trichilemmal carcinoma patients, using the log-rank test.

\begin{tabular}{|c|c|c|c|c|}
\hline Characteristics & $\begin{array}{c}\text { Cases } \\
\text { (n) }\end{array}$ & $\begin{array}{c}\text { 5-year } \\
\text { survival } \\
\text { (months) }\end{array}$ & $\chi^{2}$-value & P-value \\
\hline Gender & & & 0.253 & 0.615 \\
\hline Male & 12 & 88.9 & & \\
\hline Female & 14 & 88.9 & & \\
\hline Age (years) & & & 0.031 & 0.859 \\
\hline$>40$ & 15 & 90.9 & & \\
\hline$\leq 40$ & 11 & 87.5 & & \\
\hline Tumor size $(\mathrm{cm})$ & & & 0.027 & 0.869 \\
\hline $\mathrm{D} \leq 2.5$ & 12 & 88.9 & & \\
\hline $\mathrm{D}>2.5$ & 14 & 90.0 & & \\
\hline $\mathrm{N}$ classification & & & 22.539 & 0.000 \\
\hline $\mathrm{N}+$ & 2 & 00.0 & & \\
\hline No & 24 & 87.5 & & \\
\hline Local recurrence & & & 2.793 & 0.095 \\
\hline Yes & 12 & 77.8 & & \\
\hline No & 14 & 100 & & \\
\hline Surgery margin $(\mathrm{cm})$ & & & 8.131 & 0.004 \\
\hline$\leq 1.0$ & 10 & 71.4 & & \\
\hline$>1.0$ & 16 & 100 & & \\
\hline
\end{tabular}

$\mathrm{D}$, diameter

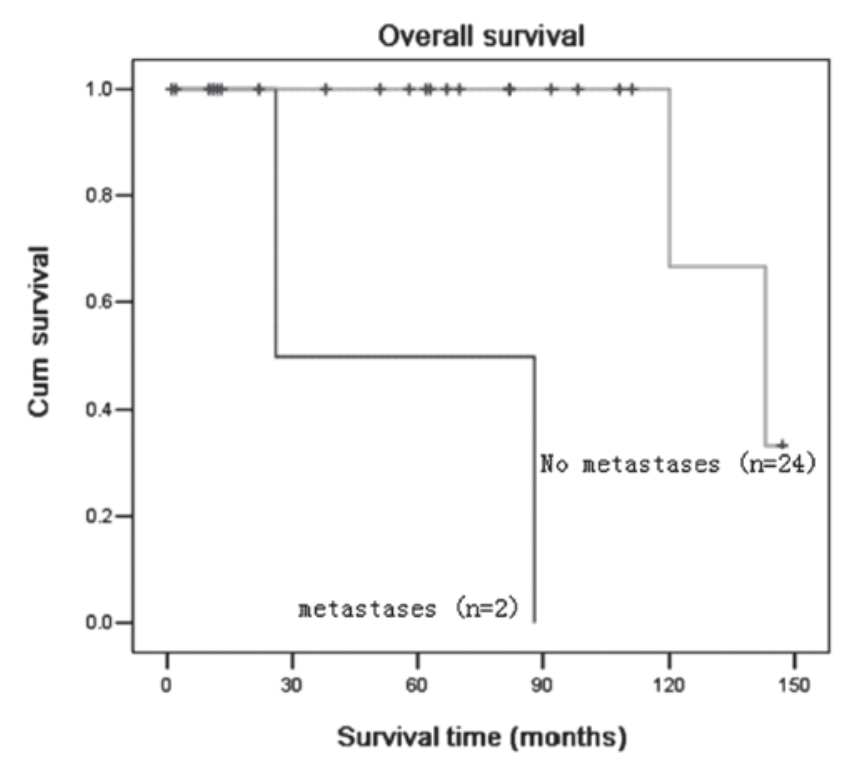

Figure 1. Kaplan-Meier curve with univariate analysis (log-rank test) for patients with distant metastases vs. patients without distant metastases against overall survival. Cum, cumulative.

is usually a solitary indolent lesion, although it can appear as multiple lesions on non-sun-exposed skin (8). Clinically, it may be misdiagnosed as squamous or basal cell carcinoma, 


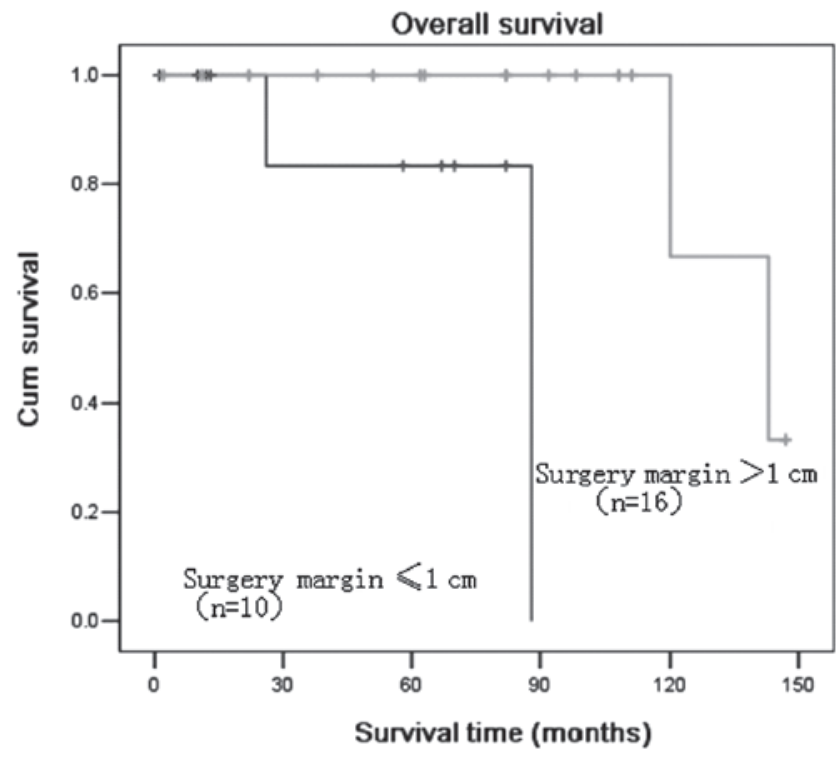

Figure 2. Kaplan-Meier curve with univariate analysis (log-rank test) for patients treated with surgery, margin $\leq 1.0 \mathrm{~cm}$ vs. patients treated with surgery, margin $>1.0 \mathrm{~cm}$ against overall survival. Cum, cumulative.

nodular melanoma or keratoacanthoma. TC has been shown to be a malignant form of a trichilemmoma (9). The diagnosis is established following histopathological examination using hematoxylin and eosin (H\&E) staining which, when necessary, is complemented by immunohistochemical staining of the lesions (10).

Generally, TC has a non-aggressive course. In their study, Boscaino et al (7) reported that after excision of the lesions in seven patients, there was no recurrence of the tumor within a 2-month to 4-year follow-up period. The clinical findings of this study were similar to those previously described $(1,3,5,7,8-10)$. First, the duration of the lesion prior to diagnosis ranged from 2 months to 50 years. In some cases, rapid growth of tumor was noted prior to presentation after a long period of time and these tumors were frequently misdiagnosed as benign lesions. Most of the TC patients had a history of significant lifetime sun exposure. The distribution of the lesions suggest that sunlight is important in the development of this malignancy (11). However, TC is an adnexal tumor, thus its regional distribution may simply reflect its appendageal origin which favors the head and neck region. Moreover, TC has been reported in burn scars, as well as in an elderly man who received 50-60 diagnostic chest radiographs for the management of pulmonary tuberculosis $(12,13)$. However, the pathogenesis of TC remains poorly understood.

Although TC is known to be an unusual malignant lesion with its histological characteristics suggesting an intermediateto high-grade malignancy, it is generally characterized by a benign neoplasm process and can be treated with complete excision (9). Routine care for this tumor has been surgical excision with the demonstration of non-invasive margins (14). Histologically, non-invasive margins are crucial for locally aggressive growth pattern and local recurrence potential $(9,14,17)$. In this study, despite the wide local excision with tumor-free margins, the tumor recurred in two cases, while $24 / 26$ patients underwent surgical excision with a satisfactory outcome. Surgery is considered to be the treatment of choice for TC, and periodic surveillance without adjuvant therapy is generally sufficient (12).

Local cervical lymph node and distant metastases of this neoplasm have rarely been reported in the medical literature $(3,9,14)$. In this study, a total of two patients succumbed to the disease one year and six months after surgery and chemotherapy (cisplatin, bleomycin and vindesine), due to regional lymph node and presented distant metastases. No optimal treatment for TC with metastases has been suggested and there is no established chemotherapy regimen reported in the medical literature. In their study, Yi et al (6) reported that a patient was treated with four cycles of cisplatin plus cyclophosphamide combination chemotherapy and a partial remission was achieved. The cisplatin plus cyclophosphamide treatment was not curative, but controlled tumor growth. In their study, Roismann et al (10) described a TC case where 4 cycles of chemotherapy (5 fluorouracil combined with cisplatin) were administered in case of poor prognosis (10). Moreover, Hayashi et al (15) and Ikegawa et al (16) administered chemotherapy [cisplatin, adriamycin and vindesine (CAV) treatment] similar to the regimen used for highly advanced cases of squamous cell carcinoma. The present study shows a recurrent TC case that was difficult to treat, which presented distant metastases $(4,6,10,15,16)$.

In summary, after the investigation of the clinicopathological characteristics of 26 TC patients, the treatment of TC is suggested to be exclusively surgical. Simple excision with $1-\mathrm{cm}$ margins is believed to be safe, cost-efficient and effective for the treatment of TC. Moreover, postoperative follow-up of the patient in order to facilitate early diagnosis of the recurrence and distant metastasis is necessary. Systemic chemotherapy should be considered when distant tumor metastasis is confirmed, which is potentially useful for the monitoring of disease progression.

\section{References}

1. Reis JP, Tellechea O, Cunha MF and Baptista AP: Trichilemmal carcinoma: a review of eight cases. J Cutan Pathol 20: 44-49, 1993.

2. Maize JC and Snider RL: Nonmelanoma skin cancers in association with seborrheic keratoses. Clinicopathologic correlations. Dermatol Surg 21: 960-962, 1995.

3. Swanson PE, Marrogi AJ, Williams DJ, Chewitz DL and Wick MR: Trichilemmal carcinoma: clinicopathologic study of 10 cases. J Cutan Pathol 19: 100-109, 1992.

4. Van Zele D, Arrese JE, Heymans O, et al: Invasive tricholemmal carcinoma of the nose. Dermatology 204: 315-317, 2002.

5. Dailey JR, Helm KF and Goldberg SH: Tricholemmal carcinoma of the eyelid. Am J Ophthalmol 115: 118-119, 1993.

6. Yi HS, Sym SJ, Park J, Cho EK, Ha SY, Shin DB and Lee JH: Recurrent and metastatic trichilemmal carcinoma of the skin over the thigh: a case report. Cancer Res Treat 42: 176-179, 2010.

7. Boscaino A, Terracciano LM, Donofrio V, et al: Tricholemmal carcinoma: a study of seven cases. J Cutan Pathol 19: 94-99, 1992.

8. Garrett AB and Scott KA: Trichilemmal carcinoma: a case report of a rare skin cancer occurring in a renal transplant patient. Transplantation 76: 1131, 2003.

9. Garrett AB, Azmi FH and Ogburia KS: Trichilemmal carcinoma: a rare cutaneous malignancy: a report of two cases. Dermatol Surg 30: 113-115, 2004.

10. Roismann M, Freitas RR, Ribeiro LC, Montenegro MF, Biasi LJ and Jung JE: Trichilemmal carcinoma: case report. An Bras Dermatol 86: 991-994, 2011. 
11. Laochumroonvorapong P, Kokta V and Quan MB: Trichilemmal carcinoma in an African American. Dermatol Surg 28: 284-286, 2002.

12. Ko T, Tada H,Hatoko M, Muramatsu T and Shirai T: Trichilemmal carcinoma developing in a burn scar: a report of two cases. J Dermatol 23: 463-468, 1996.

13. Chan KO, Lim IJ, Baladas HG and Tan WT: Multiple tumour presentation of trichilemmal carcinoma. Br J Plast Surg 52: 665-667, 1999.
14. Wong TY and Suster S: Tricholemmal carcinoma. A clinicopathologic study of 13 cases. Am J Dermatopathol 16: 463-473, 1994.

15. Hayashi I, Harada T, Muraoka M and Ishii M: Malignant proliferating trichilemmal tumour and CAV (cisplatin, adriamycin, vindesine) treatment. Br J Dermatol 150: 156-157, 2004.

16. Ikegawa S, Saida T, Obayashi H, Sasaki A, Esumi H, Ikeda S, et al: Cisplatin combination chemotherapy in squamous cell carcinoma and adenoid cystic carcinoma of the skin. J Dermatol 16: 227-230, 1989. 